\title{
Estimación teórica del efecto de la temperatura en la densidad, viscosidad, poder calorífico, capacidad calorífica y gravedad API de biocombustibles
}

\author{
Theoretical estimation of the effect of temperature on density, viscosity, calorific \\ value, heat capacity and gravity API of biofuels
}

\author{
A. A. Espitia-Cubillos iD; A. E. Delgado-Tobón iD; S. A. Camargo-Vargas (iD
}

\begin{abstract}
Resumen-El presente artículo busca llevar a cabo un análisis teórico del comportamiento de las principales propiedades del biodiesel (densidad, viscosidad, poder calorífico, capacidad calorífica, gravedad API), empleando para ello datos de la normatividad técnica mundial asociada a su producción, así como datos teóricos definidos en la literatura. A partir del análisis de las propiedades se genera una evaluación de los biodiesel en términos de su comportamiento y calidad. El desarrollo se llevó a cabo mediante la simulación de los procesos en Matlab, obteniendo un conjunto de parámetros base para la evaluación de todo biodiesel. Esta investigación emplea métodos estadísticos descriptivos, de muestreo, así como de correlación y, métodos matemáticos como mínimos cuadrados, aplicaciones lineales y exponenciales, entre otros para generar el análisis conjunto de la información, así como de los modelos empleados, logrando un mayor grado de confianza en los análisis. Los datos resultantes de la simulación para las propiedades fisicoquímicas consideradas son cercanos a los valores experimentales reportados en la bibliografía, lo que permite su uso, reduciendo los tiempos en estudios en biocombustibles.
\end{abstract}

Palabras clave- Biodiesel, Capacidad Calorífica, Densidad, Gravedad API, Poder Calorífico, Simulación, Temperatura, Viscosidad.

Abstract-The present article make a theoretical analysis of the behavior of the main properties of biodiesel (density, viscosity, calorific value, heat capacity, gravity API), using data from the global technical regulations associated with its production and theoretical data defined in the literature. From the analysis of the properties an evaluation of the biodiesel in terms of its behavior and quality is generated. The development was carried out by simulating the processes in Matlab, obtaining a set of base

Este manuscrito fue enviado el 20 de enero de 2019 y aceptado el 25 de junio de 2019. Este estudio hace parte del proyecto de investigación INV-ING2122, financiado por la Vicerrectoría de Investigaciones de la Universidad Militar Nueva Granada. Vigencia 2016.

A. A. Espitia-Cubillos, Profesora Asociada, Facultad de ingeniería, Universidad Militar Nueva Granada, Carrera 11 No. 101-80, Bogotá D. C., Colombia. (e-mail: anny.espitia@unimilitar.edu.co) parameters for the evaluation of all biodiesel. This research employs descriptive statistical methods, of sampling, as well as of correlation and, mathematical methods like least squares, linear and exponential applications, among others to generate the joint analysis of the information, as well as of the employed models, achieving a greater degree of confidence in the analyzes. Data resulting from physicochemical properties considered simulation are close to experimental values reported in bibliography, which allows their use, reducing times in biofuel studies.

Key Word - Biofuels, Calorific Power, Density, Calorific Capacity Gravity API, Simulation, Temperature, Viscosity.

\section{INTRODUCCIÓN}

T a calidad del biodiesel depende del proceso de fabricación, Udel tipo de los reactivos empleados y así mismo de la naturaleza de los lípidos de los cuales se ha producido [1]. De esta forma, la calidad del biodiesel es determinada con base en los parámetros físicos-químicos definidos en las normas técnicas, establecidas por las regiones productoras de biocombustibles.

En 2001 se expide la ley 693, articulada en la ley 939 de 2004 , constituyendo la base principal con la cual se da inicio al proceso de producción de biocombustibles en Colombia. La ley 939 de 2004 sustenta y aprueba a la fabricación y distribución de los biodiesel de diferentes fuentes (vegetal y/o animal) en motores de combustión, siguiendo las reglamentaciones para estar acorde con los requisitos de calidad dados por el Ministerio de Minas y Energía, así como el Ministerio de Medio ambiente. En la norma NTC 5444 se determina el

A. E. Delgado-Tobón, Profesor Asociado, Facultad de ingeniería, Universidad Militar Nueva Granada, Carrera 11 No. 101-80, Bogotá D. C., Colombia. (e-mail: arnoldo.delgado@unimilitar.edu.co)

S. A. Camargo-Vargas, Asistente de investigación, Facultad de ingeniería, Universidad Militar Nueva Granada, Carrera 11 No. 101-80, Bogotá D. C., Colombia. (e-mail: sacamargov@unal.edu.co) 
desarrollo de Biodiesel en motores diesel [2]. En ese documento se cuenta con los elementos y requisitos asociados a los alquíl esteres que definen los ácidos grasos, para su uso como combustible de tipo vegetal y animal, siendo estas características definidas en la NTC 1438 (para motores diesel) [3]. Desde el 1 de enero de 2008 el gobierno Colombiano establece la mercantilización del biodiesel en la mayoría del territorio y en 2003 da inicio a la Mesa Nacional de Biocombustibles (MNB), desde la cual se otorga al ICONTEC el establecimiento de las Normas Técnicas para Biocombustibles en Colombia [4].

Ahora bien, el modelado matemático como herramienta de evaluación de la calidad de los biocombustibles se ha dado desde la exploración de las propiedades físico-químicas inherentes al desarrollo de los mismos, llevando a cabo esto en la mayoría de trabajos a través de software licenciado, sin que este permita el manejo de las variables asociadas a los modelos y el análisis de datos. A través del uso de software aplicativo como HySys e ICAS entre otros, se ha simulado el proceso de obtención del biodiesel, buscando predecir los parámetros más relevantes de las diferentes Normas Técnicas [11, 30, 2], y comparando estos con datos experimentales. [5]. Esto ha logrado resultados que no permiten un proceso de análisis desde el método mismo, sin tener en cuenta la normatividad dada para los diferentes países productores y sus elementos base de producción.

Sin embargo, autores como Blanguino \& Romano [6], han estimado teóricamente valores de densidad, presión de vapor y tensión superficial para biodiesel, teniendo en cuenta valores experimentales asociados. Quezada [7], evalúo a través de cinco aceites vegetales los valores asociados a energía de combustión y algunos parámetros físico-químicos mediante el uso de software especializado (Aspen Plus).

Mediante el empleo de redes neuronales se han determinado expresiones para la viscosidad del biodiesel, empleando valores asociados a los pesos de los metil esteres [8]. No obstante, esta formulación no ha sido un buen evaluador, ya que se da a partir de un tipo de aceite y no tiene en cuenta la diversidad de metil esteres que contienen los diferentes tipos de aceite base de desarrollo de biodiesel.

Actualmente se cuenta con diversos estudios que emplean plataformas de desarrollo (fortran, Matlab, Python), que buscan evaluar los elementos básicos asociados a la fisicoquímica de los biocombustibles, como los desarrollados por Benjumea et al [9], Blanguino \& Romano [6], entre otros, esto por la falta de compilación de información y la inclusión de variables generales que permitan evaluar correctamente el biodiesel.

Teniendo en cuenta las plataformas de desarrollo como Matlab, y las falencias ya mencionadas para la evaluación de las propiedades fisicoquímicas, se propone estimar a partir de las ecuaciones de referencia, empleando la simulación, los valores propios de las variables fisicoquímicas y su comportamiento en relación a cambios de estado. Permitiendo con ello realizar aproximaciones sustentadas a valores en laboratorio, logrando así minimizar tiempos y generar mayor fiabilidad en los resultados de estudios en biocombustibles.

\section{METODOLOGÍA}

Con el propósito de conocer el comportamiento que presentan las propiedades fisicoquímicas de un biodiesel en relación con cambios térmicos, se emplearon los datos teóricos asociados a las normas que definen las propiedades y algunos otros de recopilación bibliográfica y con ello, a través del uso de Matlab y de las ecuaciones de condición de cada propiedad, simular el comportamiento y la tendencia que se tiene en relación a los cambios térmicos.

$\mathrm{Su}$ desarrollo partió de la revisión de la literatura para obtener datos de referencia de las características físicoquímicas de biodiesel obtenido mediante transesterificación, igualmente se consultó la normatividad asociada para conocer los parámetros que deben cumplirse en su producción.

Para la obtención y validación de las propiedades fisicoquímicas estudiadas se utilizó la simulación, usando la formulación de relaciones funcionales entre variables, empleando un modelo matemático asociado a cada propiedad, que permite evaluar su funcionalidad en un sistema, logrando contrastar mediciones directas e indirectas. Las relaciones suelen ajustarse a parámetros conocidos como son los datos asociados a las normas de producción de biodiesel, para tener con ello un análisis general.

Finalmente se lleva a cabo un proceso de comparación entre modelos teóricos y valores reportados en la bibliografía, para identificar tendencias y valores de referencia.

\section{RESULTADOS}

\section{A. Parámetros iniciales y condiciones generales}

La información usada se obtuvo de una recopilación de diferentes proyectos de producción de biocombustibles. Los datos asociados se caracterizan por ser biodiesel obtenidos a través de transesterificación, la cual inició con E. Duffy y J. Patrick a mitad del siglo XIX [10] este proceso es una reacción química de un aceite o grasa vegetal con un alcohol.

De igual forma se realizó un compendio de la normatividad vigente en distintos países (Tabla I y II), donde se resaltan los grandes productores de la actualidad. De las normas que actualmente rigen la calidad del biodiesel la de mayor competencia es la ASTM D6751 [11], y las normas incluidas en la Tabla I, que muestran los rangos que deben cumplir los parámetros y procesos para la elaboración de biocombustibles.

Los ácidos grasos de aceites vegetales asociados a una mayor producción de biodiesel a nivel internacional, y que han sido base de las normas europeas se muestran en la tabla 2, donde se resalta se hizo una selección por participación y correlación de los ácidos grasos presentes en todos los biodiesel derivados de aceites vegetales.

La simulación implica relaciones que se ajusten a valores conocidos, los parámetros a evaluar en la elaboración de biocombustibles son diversos, pero esta investigación ha centrado el proceso en los parámetros: Densidad, Capacidad 
TABLA I.

NORMATIVIDAD A NIVEL INTERNACIONAL ASOCIADA AL DESARROLLO Y EVALUACIÓN DE BIODIESEL

\begin{tabular}{|c|c|c|c|c|}
\hline Propiedades & Unidades & ASTM 6751 [11] & EN 14214 [30] & NTC 5444 [2] \\
\hline Densidad $\left(15^{\circ} \mathrm{C}\right)$ & $\mathrm{g} / \mathrm{mL}$ & - & $0,860-0,900$ & $0,860-0,900$ \\
\hline Número de cetano & Cetanos & Min. 47 & Min. 51 & Min. 47 \\
\hline Punto de chispa & ${ }^{\circ} \mathrm{C}$ & Min. 130 & Min. 120 & Min. 120 \\
\hline Estabilidad a la oxidación & Horas & Min 3 & Min. 6 & Min. 6 \\
\hline Índice de yodo & $\mathrm{g}-$ yodo/100 g & N.R & Máx. 120 & Máx. 120 \\
\hline Corrosión lámina de Cobre & $\mathrm{N} / \mathrm{A}$ & 1 & 1 & 1 \\
\hline Número ácido & $\mathrm{mg} \mathrm{KOH} / \mathrm{g}$ & 0,8 Máx. & 0,5 Máx. & Máx. 0,5 \\
\hline Contenido de agua & $\mathrm{mg} / \mathrm{kg}$ & 500 Máx. & 500 Máx. & 500 Máx. \\
\hline Contenido de fósforo & $\mathrm{mg} / \mathrm{kg}$ & Máx. 10 & Máx.10 & Máx. 10 \\
\hline $\begin{array}{l}\text { Contenido de metanol o } \\
\text { etanol }\end{array}$ & $\%$ masa & 0,2 & 0,2 & \\
\hline $\begin{array}{l}\text { Contenido de glicerina } \\
\text { libre/total }\end{array}$ & $\%$ masa & $0,02 / 0,24$ & $0,02 / 0,25$ & $0,02 / 0,25$ \\
\hline Contenido de éster & $\%$ masa & 96,5 & 96,5 & 96,5 \\
\hline Contenido de $\mathrm{Na}+\mathrm{K}$ & $\mathrm{mg} / \mathrm{kg}$ & Máx. 5 & Máx. 5 & Máx. 5 \\
\hline Contenido de $\mathrm{Ca}+\mathrm{Mg}$ & $\mathrm{mg} / \mathrm{kg}$ & Máx. 5 & Máx. 5 & Máx. 5 \\
\hline
\end{tabular}

TABLA II

CARACTERÍSTICAS DE LOS ÁCIDOS GRASOS CON MAYOR PRESENCIA EN LOS BIODIESEL DE ACUERDO A LA LITERATURA

\begin{tabular}{|c|c|c|c|c|c|c|}
\hline País & Alemania & Italia & Francia & Unión Europea & USA & Argentina \\
\hline Norma & DIN V 5160 & CUNA & Journal officiel & EN 14214 & ASTM D-6751 & IRAM 6515 \\
\hline Viscosidad $40^{\circ} \mathrm{C}$ cSt & $3,5-5,0$ & $3,5-5,0$ & - & $3,5-5,0$ & $1,9-6,0$ & $3,5-5,0$ \\
\hline Punto ignición ${ }^{\circ} \mathrm{C}$ & $\geq 100$ & $\geq 100$ & - & $\geq 120$ & $\geq 100$ & - \\
\hline Azufre total $\% \mathrm{P}$ & $\leq 0,01$ & $\leq 0,01$ & - & $10 \mathrm{mg} / \mathrm{kg}$ & $\leq 0,05$ & $10 \mathrm{mg} / \mathrm{kg}$ \\
\hline Destilado $10 \%$ & $\leq 0,3$ & $\leq 0,5$ & - & $\leq 0,3$ & - & $\leq 0,3$ \\
\hline Agua mg/kg & $\leq 300$ & $\leq 700$ & $\leq 200$ & $\leq 500$ & $\leq 0,05 \%$ & $\leq 500$ \\
\hline Número Cetano & $\geq 49$ & - & $\geq 49$ & $\geq 51$ & $\geq 40$ & $\geq 50$ \\
\hline $\mathrm{N}^{\circ}$ Neutralización & & & & & & \\
\hline $\mathrm{mg} \mathrm{KOH} / \mathrm{g}$ & $\leq 0,50$ & $\leq 0,50$ & $\leq 1$ & $\leq 0,50$ & $\leq 0,80$ & $\leq 0,50$ \\
\hline Metanol \%P & $\leq 0,30$ & $\leq 0,20$ & $\leq 0,1$ & $\leq 0,20$ & - & $\leq 0,20$ \\
\hline Contenido Estéres \%P & - & $\geq 98$ & $\geq 96,5$ & $\geq 96,5$ & - & $\geq 96,5$ \\
\hline Triglicérido \%P & $\leq 0,10$ & $\leq 0,10$ & - & $\leq 0,20$ & - & $\leq 0,20$ \\
\hline Diglicérido \% $\mathrm{P}$ & $\leq 0,10$ & $\leq 0,20$ & - & $\leq 0,20$ & - & $\leq 0,20$ \\
\hline Monoglicérido \%P & $\leq 0,80$ & $\leq 0,80$ & $\leq 0,80$ & $\leq 0,80$ & - & $\leq 0,80$ \\
\hline
\end{tabular}

Fuente: Autores, datos tomados de García [5], ASTM D6751 [11], Sánchez y Huertas [14] y UNE-EN 14214 [30]

TABLA III

CARACTERÍSTICAS DE LOS ÁCIDOS GRASOS EN PORCENTAJE CON MAYOR PRESENCIA EN LOS BIODIESEL DE ACUERDO A LA LITERATURA

\begin{tabular}{lcccc}
\hline \hline Nombre & Símbolo & $\begin{array}{c}\text { Peso } \\
\text { Molecular } \\
\text { Girasol }\end{array}$ & $\begin{array}{c}\text { Peso } \\
\text { Molecular } \\
\text { café }\end{array}$ & $\begin{array}{c}\text { Peso } \\
\text { Molecular } \\
\text { Micro algas }\end{array}$ \\
\hline Palmíico & $\mathrm{P}$ & 256,43 & 256 & 270,45 \\
Esteórico & $\mathrm{S}$ & 284,49 & 284 & 298,5 \\
Oleóico & $\mathrm{O}$ & 282,47 & 282 & 296,49 \\
Linoleico & $\mathrm{L}$ & 280,44 & 280 & 294,47 \\
Linolénico & LL & & \multicolumn{3}{|c}{292,46} \\
\hline \hline
\end{tabular}

calorífica, Viscosidad cinemática, Gravedad API, Poder calorífico superior y Número de Cetano, que proporcionan un análisis coherente de la preparación y obtención del biodiesel.

Sin embargo, los modelos matemáticos para algunas propiedades fisicoquímicas requieren valores que las normas no contienen, por lo que en este caso se empleó un estadístico exploratorio de valores para diferentes tipos de biodiesel logrando con ello la tabla III.

Los valores asociados al Número de Cetano, son obtenidos de diferentes fuentes, donde se resalta el aporte dado por Lujaji [12] y el dado por Knothe et al, [13], estos valores se encuentran
TABLA IV

VALORES PROMEDIO DE NUMERO CENTANO (CN) PARA LOS ÁCIDOS GRASOS CON PRESENCIA EN LOS BIOCOMBUSTIBLES OBTENIDA DE LA LITERATURA

\begin{tabular}{cc}
\hline \hline Ácidos Grasos & Promedio \\
\hline Esteárico & 86 \\
Palmítico & 76,7 \\
Mirístico & 70 \\
Laúrico & 61 \\
Oleico & 57 \\
Linoleico & 39.3 \\
Linolénico & 27,8 \\
\hline \hline
\end{tabular}

Fuente: Autores a partir de Lujaji [12] y Knothe [13]

asociados a generación de biodiesel a partir de aceites vegetales y frutos de semilla a través el proceso de transesterificación, las tablas IV y V muestran los ácidos grasos que presentan mayor relación con los aceites vegetales base para la producción de biodiesel, así como los de mayor peso molecular en la obtención del mismo.

\section{B. Evaluación de Densidad}

La densidad proporciona una idea del contenido en energía en un combustible. Altas densidades son un indicador de mayor energía térmica, por lo tanto, un funcionamiento de 
combustible mejor, más se ha demostrado que la mayor densidad del biodiesel puede causar problemas en los actuales sistemas de inyección de los motores y, a bajas temperaturas, plantea problemas de solidificación y congelación [15].

En el Sistema Internacional se mide en $\mathrm{kg} / \mathrm{m} 3$. En los líquidos el volumen varía con la temperatura y disminuye con la presión, así mismo cambia la densidad por lo que debe especificarse la temperatura con la que se mide.

La construcción de la curva de densidad ( $\rho$ ) para el biodiesel se trabajó teniendo en cuenta el rango de temperaturas dadas por las normas y los valores fisicoquímicos provistos en las mismas (tablas I y II), además de datos de literatura, obteniéndose la curva (Fig. 1), a partir de (1).

$$
\rho=\frac{\rho_{R}}{\left(Z_{R A}\right)^{\phi}}
$$

En este caso $\rho_{R}$ es la densidad experimental $(\mathrm{kg} / \mathrm{m} 3)$ evaluada a una temperatura de referencia $\left(T_{R}\right)$, siendo la referencia dada próxima a $273^{\circ} \mathrm{K}, \mathrm{Z}_{\mathrm{RA}}$ es un valor constante para cada compuesto y $\phi$ se obtiene mediante (2):

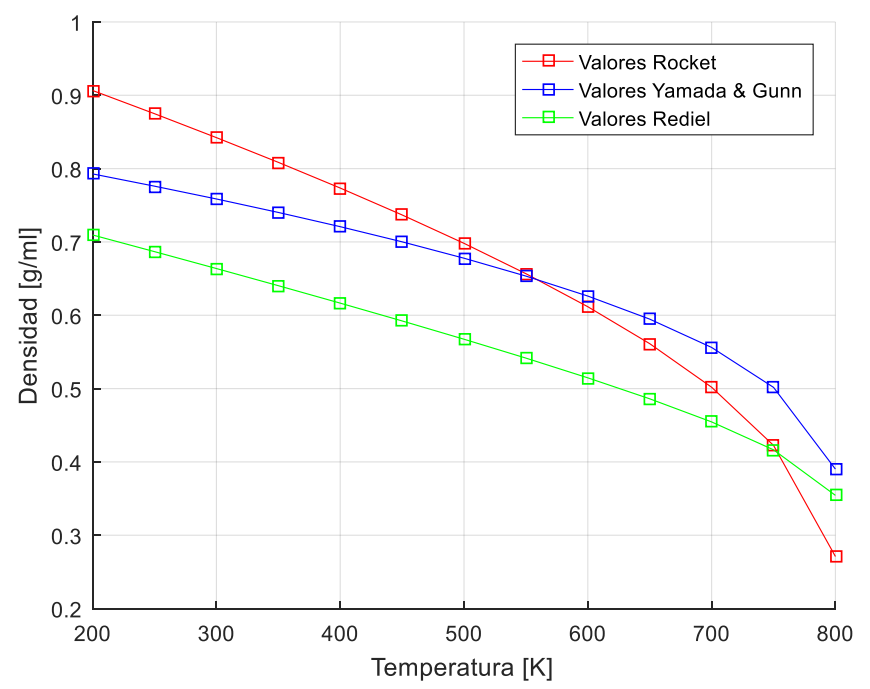

Fig. 1. Evaluación de modelos teóricos de densidad en relación con la temperatura.

$$
\phi=\left(1-\frac{T}{T_{c}}\right)^{\frac{2}{7}}-\left(1-\frac{T_{R}}{T_{c}}\right)^{\frac{2}{7}}
$$

El valor de $\mathrm{T}$ es determinado a partir del promedio de los valores máximos y mínimos definidos en las normas asociadas a la producción de biocombustible (tablas I y II), así como a valores obtenidos de los estudios realizados por Blanguino \& Romano [6], Quezada [7] y Castro [15].

Se evaluaron dos modelos empíricos que se describen a continuación, que se estimaron teniendo en cuenta su aplicabilidad en el análisis de fluidos y valores de temperatura de rangos y características similares a los del Biodiesel.
La expresión más sencilla fue la propuesta por Yamada y Gunn [16], y esta se muestra en (3):

$$
\rho=\left[V_{c}(0.29056-0.08775 \omega)^{\left(1-\frac{T}{T_{c}}\right)^{\frac{2}{7}}}\right]^{-1}
$$

En (3), $V_{c}$ es el volumen crítico evaluado desde el promedio de los datos definidos en la bibliografia $[6,7,15]$, para diferentes tipos de Biodiesel basados en semillas, el valor del factor acéntrico $\omega$ (adimensional) se obtiene mediante la formulación empírica (4) definida por Reid [17]:

$$
\omega_{i}=\frac{3}{7}\left[\frac{\theta}{(1-\theta)}\right] \log P_{c}-1
$$

Donde se cuenta con una variable $\theta$ (5), que relaciona los valores de la temperatura de ebullición de $\mathrm{T}_{\mathrm{b}}$ y la temperatura critica $\mathrm{T}_{\mathrm{c}}$, ambas en grados Kelvins (K). Así mismo se cuenta con la presión crítica $\mathrm{P}_{\mathrm{c}}$ (bar), evaluada desde las normas colombianas y las internacionales.

$$
\theta=\frac{T_{b}}{T_{c}}
$$

La expresión dada por Riedel [18], es una modificación a la mencionada por Yamada y Gunn [16], esta es evaluada y modificada a partir de los resultados experimentales en diferentes fluidos asociados a biocombustibles y se presenta en (6).

$$
\rho=\frac{1}{V_{c}}\left[1+0.85\left(1-\frac{T}{T_{c}}\right)+(1.6916+0.984 \omega)\left(1-\frac{T}{T_{c}}\right)^{\frac{1}{3}}\right]
$$

\section{Evaluación de la relación Viscosidad - Temperatura}

La viscosidad de un fluido de tipo newtoniano no es dependiente del tiempo en el cual se tenga una aplicación de esfuerzo, aunque sí puede asociarse tanto a la temperatura como a la presión a la que se encuentre. La relación de la temperatura con la viscosidad cinemática se estudió en un rango de temperaturas que oscila en valores de 16 a $100{ }^{\circ} \mathrm{C}$, evaluando en intervalos de $10^{\circ} \mathrm{C}$. De acuerdo con Kerschbaum et al [19], el biodiesel pierde su naturaleza Newtoniana como consecuencia de la formación de elementos cristalinos a temperaturas inferiores a $\operatorname{los} 16^{\circ} \mathrm{C}$.

Se emplearon valores de viscosidad asociados a las normas técnicas referidas en las tablas I y II, para contrastar, estimando desde las fórmulas de extrapolación el comportamiento teniendo en cuenta a Souders [5] entre otros, comparando así los valores generados y su proximidad a las diferentes normas.

TABLA V

COMPOSICIÓN DE LOS ÁCIDOS GRASOS ASOCIADOS A DIFERENTES TIPOS

\begin{tabular}{cccccc}
\multicolumn{5}{c}{ DE BIODIESEL } \\
\hline \hline Biodiesel & $16: 0$ & $18: 0$ & $18: 1$ & $18: 2$ & $\mathbf{1 8 : 3}$ \\
\hline Palma & 46,7 & 3,7 & 39,6 & 8,4 & 0,2 \\
Soya & 13,9 & 2,1 & 23,1 & 56,3 & 4,2 \\
Colza & 4,4 & 1.2 & 65,2 & 21 & \\
Girasol & 5,6 & 4,1 & 24,3 & 65,6 & \\
Jatropha & 14,9 & 9,5 & 40,5 & 34,7 & 7 \\
Algodón & 20 & 2,8 & 19,2 & 55,4 & 0,6 \\
Karanja & 10 & 7,8 & 53,3 & 19 & 0,3 \\
\hline \hline
\end{tabular}

Fuente: Autores a partir de Garcia [5], Lujaji [12] y Knothe[13] 
El método de Lewis - Squires (7), se emplea cuando se tiene un solo dato como referencia de viscosidad y se busca evaluar el comportamiento de un valor nuevo en un intervalo de temperatura. Es así como de acuerdo con un análisis de datos obtenidos de fuentes como Cheenkachorn [8] y Benjumea [9] entre otros, en los que se emplea el método, se ha determinado que el error de predicción es próximo al 5\%, teniendo presente que el método no es aplicable a valores base que se encuentren muy por encima de punto de ebullición del fluido evaluado.

$$
\mu^{\wedge}(-0.2661)=\mu \_1^{\wedge}(-0.2661)+\left(T-T \_1\right) / 37.073
$$

De acuerdo con las normas que definen los valores de calidad de los biocombustibles a nivel mundial y teniendo como base un valor de temperatura $\left(\mathrm{T}_{1}\right)$ de $40^{\circ} \mathrm{C}$, se escogieron diversos valores de

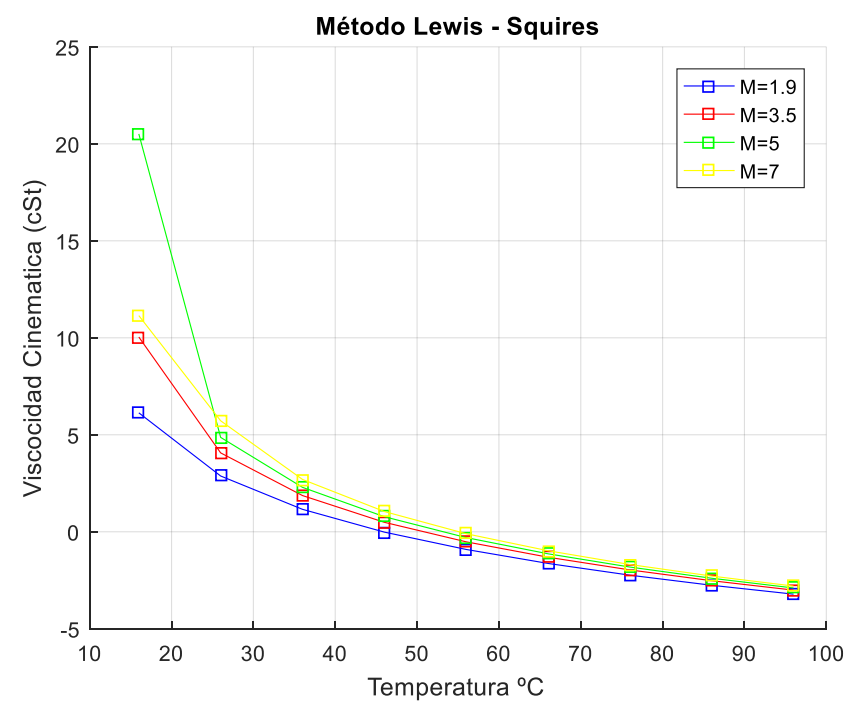

Fig 2. Aplicación del método de Lewis - Squires para el cálculo de viscosidad cinemática en relación a los cambios de temperatura, donde $\mathrm{M}$ representa los valores de viscosidad base $\left(\mu_{1}\right)$ en $\mathrm{Pa} \cdot S$

viscosidad base $\left(\mu_{1}\right)$ en $P a \cdot s$, con los que se evaluó los resultados que son posibles obtener (Fig. 2), teniendo la viscosidad Cinemática en unidades de Stokes, dado que se define desde la viscosidad dinámica.

\section{Poder Calorífico}

El poder calorífico es una propiedad importante que caracteriza el contenido energético de un combustible, ya sea sólido, líquido o gaseoso; esta propiedad es especialmente importante para los aceites vegetales y el biodiesel. Los autores Channiwala \& Parikh

TABLA VI

ECUACIONES DE CÁLCULO DE PODER CALORÍFICO OBTENIDAS DE LA LITERATURA

\begin{tabular}{cc}
\hline \hline Autor & Ecuación \\
\hline Dulong & $H H V=0,3383 C+1443\left(H-\left(\frac{O}{8}\right)\right)+0,9942 S$ \\
{$[25]$} & \\
Vondrecek & $H H V=(0,373-0,00026 C) C+1,444\left(H-\left(\frac{1}{10}\right) O\right)$ \\
{$[25]$} & $+0,1047 S$ \\
& $H H V=0,341 C+1,323 H+0,0685-0,0153 A$ \\
IGT & $-0,1194(O+N)$ \\
{$[25]$} & \\
\hline \hline
\end{tabular}

Fuente: Autores a partir de [25]
[20], mostraron una recopilación de modelos propuestos por diferentes autores, en metodologías de cálculo del poder calorífico estando esto expresado en $\mathrm{MJ} / \mathrm{kg}$ y las composiciones en porcentaje másico, parte de esta recopilación se puede observar en la Tabla 6 .

Esta estimación se produjo a través de la comparación de los valores recolectados de autores como Vivas [1], García [5], Blanguino \& Romano [6], Abdalla [10], y ASTM D6751 [11], entre otros, teniendo presente el cumplimiento de rangos teóricos y de las mismas fuentes con el fin de comprender el comportamiento de un biodiesel de acuerdo a su composición química, esto se evaluó empleando MatLab para el desarrollo de las ecuaciones, teniendo presente que de los modelos consultados solo se valoraron los parámetros definidos en la metodología (Densidad, Capacidad calorífica, Viscosidad cinemática, Gravedad API, Poder calorífico superior y Número de Cetano), debido a la información disponible en la red y los resultados teóricos que arrojaron en una primera etapa donde solo esos parámetros mostraron ser consistentes con resultados obtenidos en trabajos similares.

Los valores incluidos en las tablas I, II y III se emplean para evaluar los diferentes modelos, se observa en la Fig. 3 que los

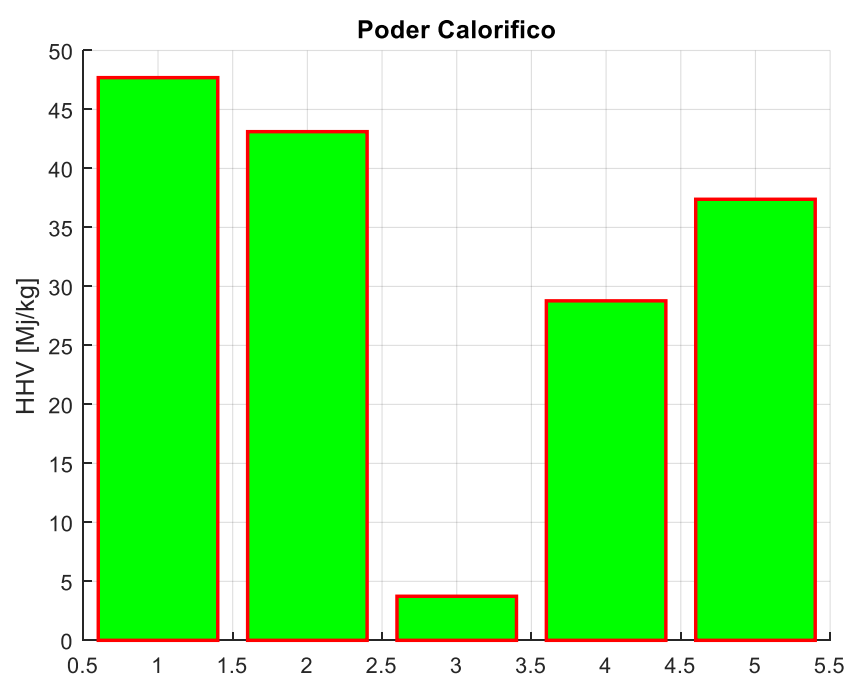

Fig 3. Estimación de valores a partir de los modelos teóricos dados en la tabla 6. (Valores enteros corresponden a los siguientes modelos: 1. Dulong, 2. Vondrecek, 3. Schuster, 4. Jenkis y 5. Beckam)

desarrollados por Dulong (1) y Vondrecek (2) muestran un comportamiento similar, con una diferencia inferior a $2 \mathrm{Mj} / \mathrm{kg}$, mientras que los modelos generados desde Jenkins (4) y Beckman (5) presentan una diferencia de $7 \mathrm{Mj} / \mathrm{kg}$ entre ellos, y de aproximadamente $10 \mathrm{Mj} / \mathrm{kg}$ respecto a los modelos (1) y (2). Los valores del modelo de Schuster (3) no tienen coherencia con los valores teóricos y ni los dados por la literatura.

El poder calorífico en términos de la temperatura se relaciona desde la formulación dada por Demirbas [21], donde se emplea la Viscosidad como elemento base del cálculo, siendo en este caso empleado el modelo ya mencionado de Lewis -Squire para la viscosidad, esto mediante (8):

$$
P C=(0,4625 * \mu)+39,450
$$


Donde las constantes dadas en la ecuación lineal (8): punto de corte y pendiente, son obtenidas mediante pruebas de

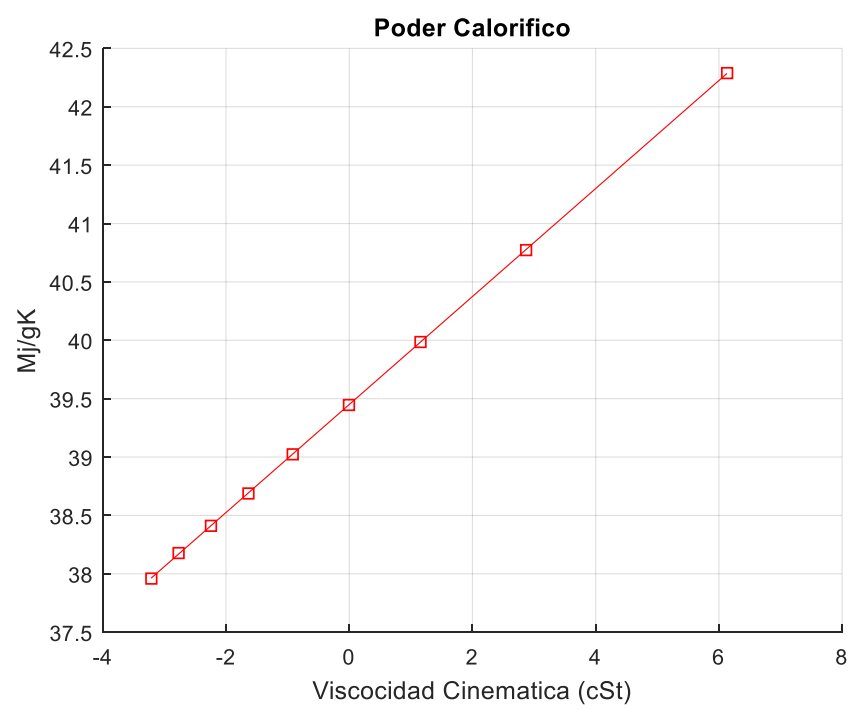

Fig 4. Estimación del Poder Calorífico a partir de Viscosidad Cinemática obtenida por el método de Lewis-Squires

laboratorio en diferentes tipos de biocombustibles, logrando un proceso coherente. El resultado (Fig. 4) evidencia una relación directa entre el poder calorífico y la viscosidad de los biodiesel, dado que entre más viscosidad se tenga, más poder calorífico ha de tener el compuesto.

Desde el uso del método de Lewis-Squires en relación de Viscosidad y temperatura, se hizo una estimación matemática de la variación que pudiese tener el Poder Calorífico de acuerdo a las variaciones de la temperatura (Fig. 5), observando un comportamiento exponencial, donde a medida que la temperatura aumenta, el poder calorífico disminuye. Esto se puede relacionar

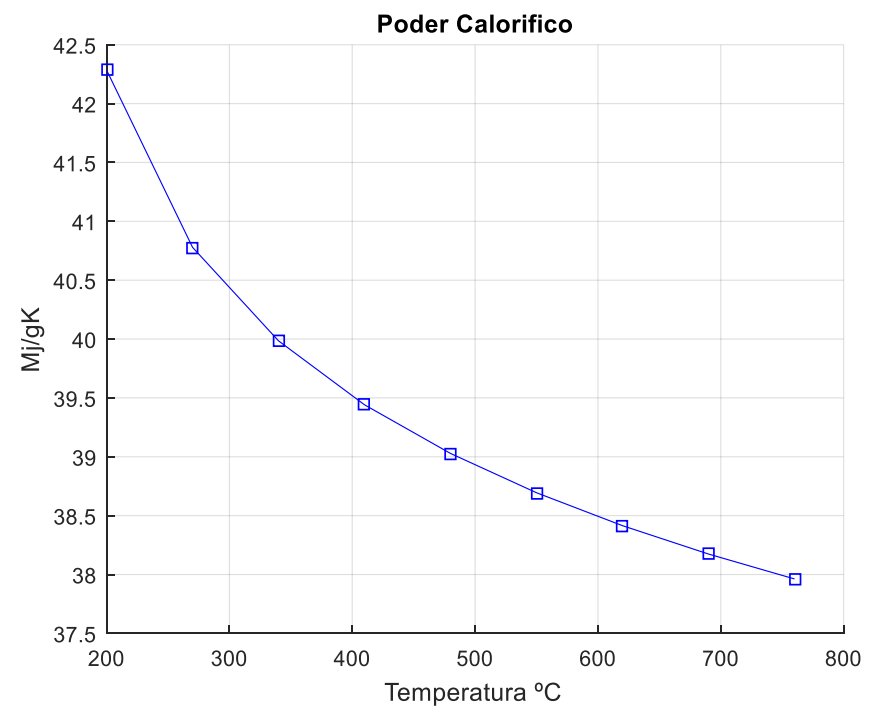

Fig 5. Estimación del Poder Calorífico en relación con la Viscosidad de LewisSquires, mostrando su variación con la temperatura.

con los elementos que componen el biodiesel (ácidos grasos), dado que puede darse cambios sustanciales si se emplea aceites derivados de semillas, algas o derivados de animales, sin embargo la curva tiene un comportamiento similar a lo identificado por García [1], Rediel [18] y Demirbas [21].

\section{E. Capacidad Calorífica}

La capacidad calorífica para algunos líquidos se encuentra en función de la temperatura a través de (9):

$$
C P=\left(A+B T-C T^{2}+D T^{3}\right) * 4,187
$$

Las constantes A, B, C y D de (9) se encuentran tabuladas para algunos líquidos en la literatura, siendo obtenidas a partir de mínimos cuadrados, empleando (9) con valores teóricos y pruebas de laboratorio, sin embargo, en este caso se usaron las siguientes tomadas de Reid [17] y Valencia [22]:

$A=1,481 ; B=15,56 \times 10^{-3} ; C=43,7 \times 10^{-6} ; D=44,09 \times 10^{-9}$

La ecuación (9) se utilizó para calcular la capacidad calorífica del Biodiesel en función de la temperatura. Cuando no se conoce o no está disponible el valor de la capacidad calorífica de los líquidos, para líquidos de naturaleza orgánica. Valencia [22], propone (10), esta da la relación entre la capacidad calorífica en unidades másicas y la masa molecular, a una temperatura de $25{ }^{\circ} \mathrm{C}$, con bastante exactitud:

$$
C P=r M^{a}
$$

Donde $\mathrm{M}$ es la masa molecular y $\mathbf{r}$ y a son constantes que dependen de la familia a la que pertenece el compuesto. Para ésteres $\mathbf{r}$ ha sido estimado como 0,60; y a como $-0,0573$. Anand [23] correlacionó el peso molecular de varios metilésteres (M), con su capacidad calorífica y propone (11):

$$
C P=-0.000001 M^{2}+0.0009 M+1.9291
$$

Para las mezclas, la estimación de calores específicos se puede hacer en forma aproximada asumiendo mezcla ideal. Aunque esto no es cierto, particularmente para los electrolitos, se obtienen estimaciones razonables.

De los modelos mencionados se pudo hacer una evaluación de $\mathrm{CP}$, a partir de (9), donde se observa que con el aumento de la temperatura la $\mathrm{CP}$ también aumenta, teniendo para una temperatura base $\left(200^{\circ} \mathrm{K}\right)$ un valor de $0,9 \mathrm{~J} / \mathrm{gk}$, sin que se observe una silla el modelo obtenido (Fig. 6), evidenciando un incremento constante de la CP con la temperatura. Se emplean para la construcción del modelo, los datos proporcionados en la literatura y en las normas (Tablas 1 a 2), proporcionando así un modelo general a los diferentes tipos de biodiesel que se generan.

Es conocido que el $\mathrm{CN}$ depende fundamentalmente de la composición química del combustible analizado [24]. El CN mide la calidad de ignición de un combustible diesel. Un alto $\mathrm{CN}$ indica un menor retraso en la ignición, ayuda a un mejor arranque del motor y minimiza la emisión de humo al lograr una combustión más suave [25]. El índice de cetano es más favorable en el biodiesel y esta ventaja se hace mayor en metilésteres con mayor porcentaje de ácidos saturados. Cheenkachorn [8] propone (12) para estimar el número de 
cetano asociado al biodiesel y que se da en función de su contenido de metilésteres.

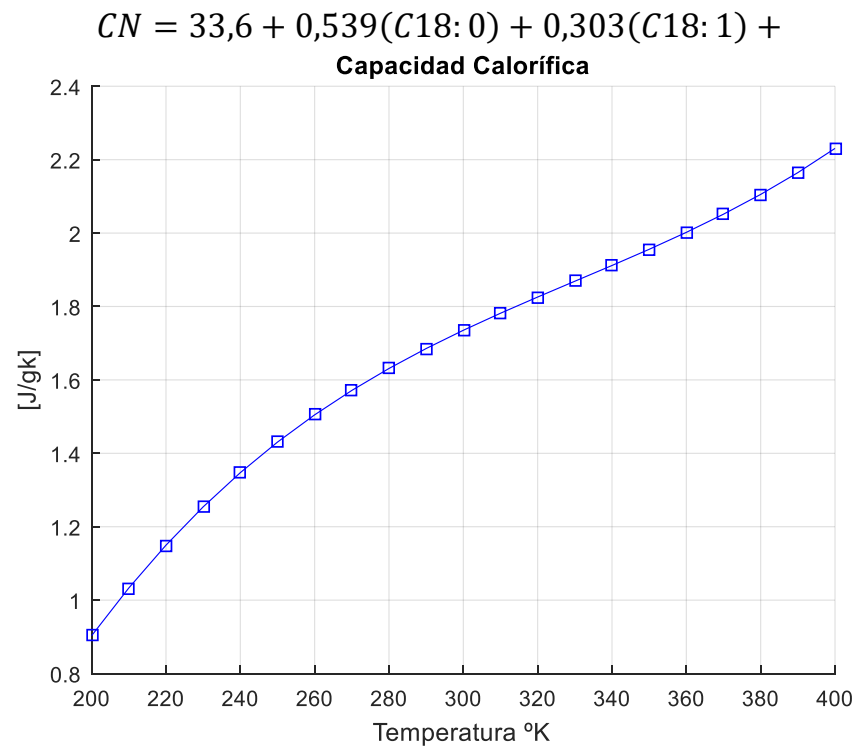

Fig. 6. Fig 6. Estimación de la Capacidad Calórica a través de un modelo polinomial de tercer orden

$$
0,0878(C 18: 2)+0,233(C 22: 1)
$$

Mediante un análisis de valores dados por la literatura, Barroto et al [24], define el modelo más ajustado (13), teniendo presente para ello un total de 16 aceites fuente, y un total de 8 ácidos grasos asociados a estos aceites, mostrando una correlación alta entre los valores de los diferentes biodiesel.

$$
\begin{gathered}
C N=6,622+0,266 L L+0,330 L l+0,5030+0,686 P+ \\
0,792 S
\end{gathered}
$$

A partir de la base de datos obtenida (Tabla V), se hizo uso de los modelos de regresión múltiple (MRM) para la evaluación de valores de $\mathrm{CN}$ asociados a los diferentes biodiesel, lográndose con ello un análisis de significancia de los ácidos grasos presentes en los biocombustibles. (Tabla VII)

TABLA VII

VALORES ESTADÍSTICOS OBTENIDOS A TRAVÉS DEL SOFTWARE R PARA EL MODELO DE REGRESIÓN CON INTERCEPTO

\begin{tabular}{lllll}
\hline \hline & Coeficiente & Error típico & $\begin{array}{c}\text { Estadístico } \\
\mathrm{t}\end{array}$ & Probabilidad \\
\hline Intercepción & 31,0512761 & 40,3663967 & 0,769235 & 0,58256 \\
$\mathrm{P}$ & $-0,3484452$ & 0,38207271 & $-0,911986$ & 0,529284 \\
$\mathrm{~S}$ & $-0,21588687$ & 0,68141881 & $-0,316819$ & 0,804675 \\
$\mathrm{O}$ & $-0,25729386$ & 0,48738186 & $-0,527910$ & 0,690777 \\
$\mathrm{~L}$ & $-0,25143772$ & 0,4098576 & $-0,61347$ & 0,649687 \\
$\mathrm{LL}$ & $-0,66176556$ & 0,88060548 & $-0,751489$ & 0,589728 \\
\hline \hline
\end{tabular}

A través del análisis de MRM, se evidencia que el uso del valor constante o intercepto genera un cambio significativo en el modelo, ya que el coeficiente de determinación aumenta, logrando con ello explicar de una manera apropiada la correlación existente entre las variables definidas mediante (14).

$$
\begin{gathered}
C N=31,051+0,348 P+0,215 S+0,2570+0,251 L- \\
0,661 L L
\end{gathered}
$$

El coeficiente de determinación $\mathrm{R}^{2}$ asociado al resultado del MRM muestra un valor de 0.777 , lo que resulta en un modelo de fiabilidad.

El intervalo dado en la predicción de valores usando la ecuación anterior, se excede en límites de valores iniciales (Fig. 7), siendo esta evidencia junto con la dada por el valor de $\mathrm{R}^{2}$, elementos que explican la variabilidad de la variable dependiente en no más de un $65 \%$.

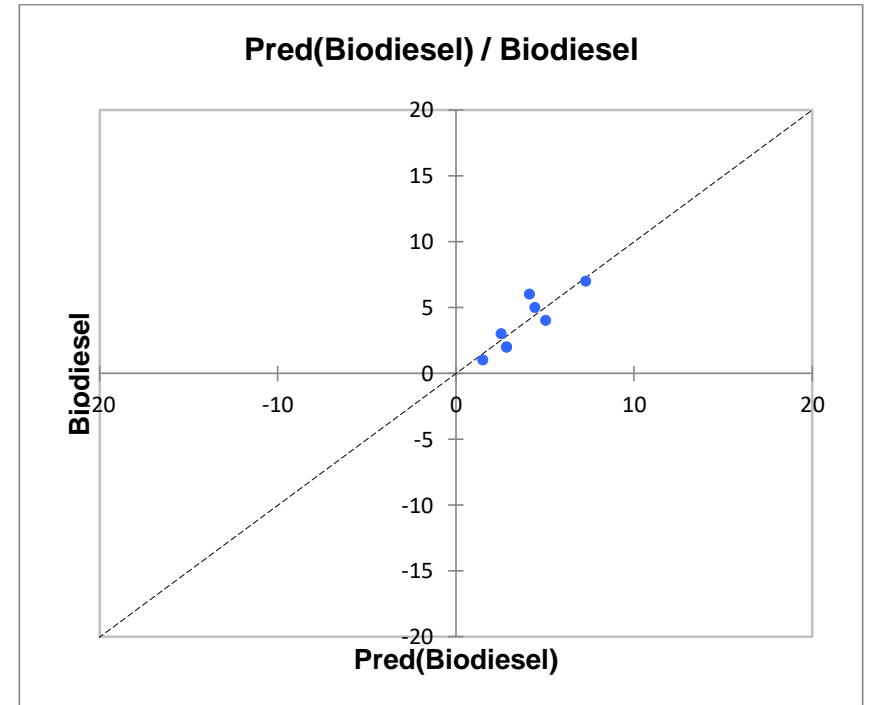

Fig. 7. Modelo de Regresión Lineal con intercepto para la predicción de valores de $\mathrm{CN}$ en Biodiesel

Se eliminó el término del intercepto en el modelo inicial, como se muestra en la Tabla VIII, lo que representó un aumento en el coeficiente $\mathrm{R}^{2}$, teniéndose 0,929 para este caso, lo que implica una mejor relación de los ácidos grasos en el compuesto (Fig. 8).

TABLA VIII

VALORES ESTADÍSTICOS OBTENIDOS PARA EL MODELO DE REGRESIÓN SIN INTERCEPTO

\begin{tabular}{lllll} 
& & \multicolumn{3}{c}{ REGRESIÓN SIN INTERCEPTO } \\
\hline \hline & Coeficiente & Error típico & Estadístico t & Probabilidad \\
\hline $\mathrm{P}$ & $-0,05953172$ & 0,06254582 & $-0,95180968$ & 0,44164981 \\
$\mathrm{~S}$ & $-0,025368$ & 0,16632512 & $-0,04479407$ & 0,96834169 \\
$\mathrm{O}$ & 0,10922428 & 0,09148795 & 1,19386512 & 0,35493263 \\
$\mathrm{~L}$ & 0,06213502 & 0,03796791 & 1,63651406 & 0,24337383 \\
$\mathrm{LL}$ & $-0,73264024$ & 0,78128588 & $-0,93773645$ & 0,4473711 \\
\hline \hline
\end{tabular}

La ecuación (15) representa el modelo finalmente obtenido el cual muestra que el valor de probabilidad para cada elemento es inferior a 1 , lo que aduce una significancia estadística, aun cuando es alto, teniendo presente un nivel de confianza cercano al 95\%.

$C N=0,858 P+0,742 S+0,5610+0,427 L+0,322 L L$

El modelo (15) muestra una mejora en el comportamiento de las variables, pues se logra una explicación de la variable dependiente en algo más de un $80 \%$. El criterio de aceptación se basó en la varianza residual buscando la sumatoria mínima. 
Evaluando los diferentes modelos expuestos con anterioridad y teniendo en cuenta los valores promedio de los biodiesel, se pudo determinar que el proceso usado evidencia un nivel de confianza suficiente.

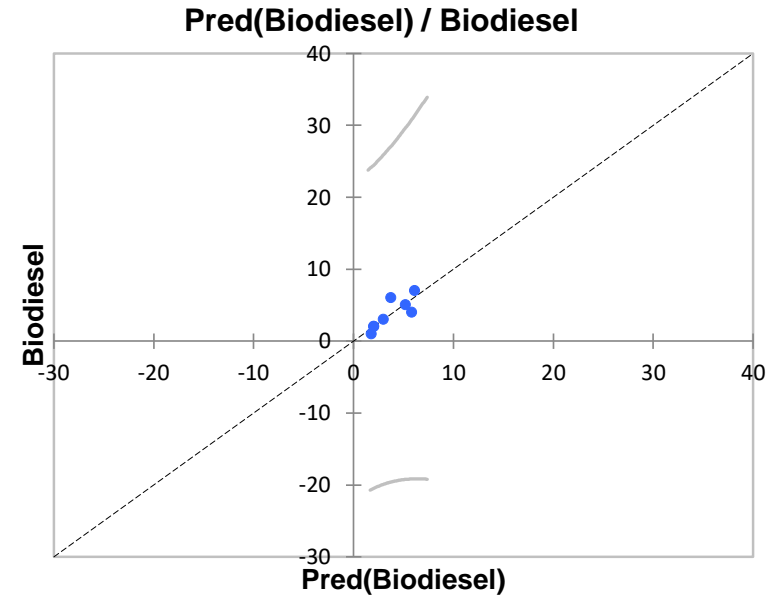

Fig. 8. Modelo de Regresión Lineal sin intercepto para la predicción de valores de $\mathrm{CN}$ en Biodiesel

Los modelos teóricos con los que se concibe la mayoría de los datos muestran una coherencia como lo evidencia el modelo generado por Barroto et al. [24], donde toma un modelo teórico y lo modifica de acuerdo a valores recolectados en la literatura. El análisis de los datos en este proyecto arrojo dos modelos dados a través de un proceso de regresión múltiple y en cual se excluyó para el segundo modelo el intercepto de la función. Se evidencia en la Tabla IX, que el modelo teórico modificado presenta una diferencia no mayor 0,4 respecto de los valores promedio encontrados en la literatura, esto contrasta con lo definido por los modelos obtenidos en este proyecto donde el modelo inicial (con intercepto) muestra una diferencia cercana a 7 y el modelo final una diferencia de 8.4. Sin embargo, el modelo final muestra, a diferencia del modelo inicial, un mejor ajuste en la mayoría de los valores, dando como valor anómalo el asociado al biodiesel desarrollado por aceite de palma, pero explicando el resto de biodiesel de forma adecuada.

TABLA IX

CUADRO COMPARATIVO DE LOS VALORES DE CN ASOCIADOS A DIFERENTES TIPOS DE BIODIESEL EVALUADOS CON TRES MODELOS DIFERENTES

\begin{tabular}{lcccc}
\hline \hline \multirow{2}{*}{ Biodiesel } & $\begin{array}{c}\text { Valor } \\
\text { Literatura }\end{array}$ & $\begin{array}{c}\text { Modelo } \\
\text { Barroto }\end{array}$ & $\begin{array}{c}\text { Modelo } \\
\text { Inicial }\end{array}$ & $\begin{array}{c}\text { Modelo } \\
\text { Final }\end{array}$ \\
\hline Palma & 61,6 & 63,3326 & 60,251 & 68,680 \\
Soya & 45 & 49,1361 & 53,631 & 51,836 \\
Colza & 53,2 & 52,1784 & 50,240 & 52,463 \\
Girasol & 50,3 & 47,7413 & 56,195 & 49,683 \\
Jatropha & 52,2 & 56,2697 & 57,198 & 57,467 \\
Algodón & 51,6 & 50,6854 & 56,990 & 53,890 \\
Karanja & 52,5 & 52,7671 & 54,609 & 52,414 \\
\hline \hline
\end{tabular}

\section{F. Gravedad API}

La gravedad API es una medida del peso de un combustible en relación al agua. $\mathrm{Si}$ el combustible es más liviano que el agua su grado API es mayor de 10. La gravedad API se puede calcular a partir de los datos de densidad, de acuerdo (16):

$$
A P I=\frac{141,5}{\rho}-131,5
$$

Donde $\rho$ es la densidad $\left(\mathrm{kg} / \mathrm{m}^{3}\right)$, evaluada desde los modelos ya presentados y con los cuales se puede obtener la variabilidad de la gravedad API en función del tiempo. La Fig. 9 presenta los resultados obtenidos.

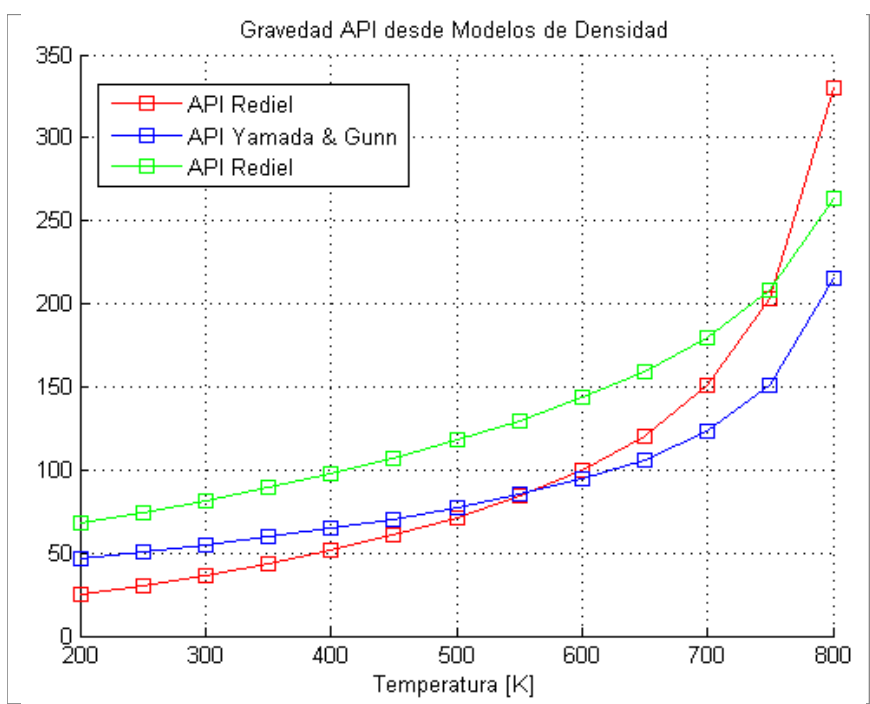

Fig. 9. Estimación de la Gravedad API a partir de los valores obtenidos en diferentes modelos de cálculo de densidad, teniendo presente la variación de la misma con la temperatura.

\section{DISCUSIÓN Y CONCLUSIONES}

Debido a que los biodiesel dependen de diferentes componentes (ácidos grasos) y procesos de desarrollo, se han generado normatividades que se acoplan a sus elementos y además las zonas de producción de los mismos (Tablas I y II), sin embargo se evaluaron elementos comunes a las normas y a la literatura generando con ello un con contraste de datos que estadísticamente proporciono un grado mayor de fiabilidad.

La Fig. 1, muestra el efecto de la temperatura en la densidad, donde el rango de temperatura evaluado comprende entre $200 \mathrm{~K}$ a $800 \mathrm{~K}$, ya que las ecuaciones empleadas no evidencian un comportamiento lógico a partir de esta temperatura. Los modelos empleados para la evaluación de la densidad (Fig. 2), muestran que la densidad disminuye a medida que se acercan al punto de ebullición, teniendo a temperaturas cercanas a los $200 \mathrm{~K}$ valores entre $0,9 \mathrm{~g} / \mathrm{mL}$ y $0,7 \mathrm{~g} / \mathrm{mL}$ y a temperaturas altas cercanas a los 800 $\mathrm{K}$ valores entre $\operatorname{los} 0,4 \mathrm{~g} / \mathrm{mL}$ a $0,3 \mathrm{~g} / \mathrm{mL}$, lo que puede darse debido a la expansión del líquido por el efecto térmico.

Los modelos empleados para la evaluación de la densidad muestran que a altas temperaturas no hay diferencias significativas en el comportamiento del biocombustible teóricamente dado en las normas técnicas, sin embargo es importante observar en la Fig. 1, que los modelos de Yamada-Gunn [16] y el de Rediel [18], evidencian una mayor estabilidad respecto del establecido por Rockett citado por Anand [23] y Benavides [26], que decae de 
forma más rápida, aun cuando autores como Rivera [25], le adjudican a este último modelo un grado alto de confianza, por lo tanto y teniendo en cuenta estudios como los de Quezada [7] y Castro [15], es ideal el empleo de los modelos de Yamada-Gunn [16] y el de Rediel [18], para la evaluación de la densidad.

Diferentes métodos de estimación de la viscosidad se encuentran en la literatura, más se hace relevante para este caso el exponer solo aquellos que pudiesen ser evaluados desde los datos asociados a las normas técnicas (tablas 1 y 2) y que a su vez puedan valorar desde los cambios en temperatura, es por esto que se emplea el modelo de Lewis - Squires, el cual evalúa a partir de unas viscosidad dinámica inicial tomada de las normas (tablas I y II), los valores de una viscosidad cinemática, como se observa en la Fig. 2.

El modelo de Lewis - Squires (Fig. 2), expone que a temperaturas bajas la viscosidad cinemática varía entre 5 y $20 \mathrm{cSt}$ hasta alcanzar valores por debajo de cero a temperaturas superiores a $\operatorname{los} 50^{\circ} \mathrm{C}$. Es evidente en la Fig. 2, que el método aunque es uno de los más usados en el estudio de biodiesel, presenta una anomalía al tomar valores cercanos a 5 en la densidad dinámica (valor de entrada del modelo), sin embargo el modelo responde correctamente a la extrapolación de valores en comparación con los modelos basados en la fracción de la mezcla como el dado por la regla de Grunberg \& Nissan [27] y trabajado por Caballero [28], mostrando a valores de $40^{\circ} \mathrm{C}([2,3,4,29,30])$ valores de viscosidad cinemática que oscilan entre 3.5 y $5 \mathrm{cSt}$.

Para la evaluación del contenido energético del biodiesel, se emplearon 5 modelos iniciales (Fig. 3), donde el modelo realizado por Schuster no explica coherentemente lo encontrado en la literatura, ni lo expuesto por las normas técnicas (tablas I y II). La Fig. 4 muestra que el poder calorífico se incrementa linealmente a medida que aumenta la viscosidad cinemática y disminuye al aumentar la temperatura (Fig. 5), lo que es coherente con la definición de poder calorífico y con los valores dados en la literatura, así como los asociados a las normas técnicas (tablas I y II).

Lo anterior permite inferir que el modelo llevado a cabo por Demirbas [21] y relacionado con el modelo de Lewis - Squieres explica el proceso de pérdida o potencial energético asociado a un biodiesel.

La capacidad calórica tiene en cuenta los metil esteres empleados en la consecución del biodiesel, es así como dada la información desde las normas técnicas y lo encontrado en la literatura, el modelo polinomial es el más acertado (Fig. 6), logrando tener valores similares a los dados por Dzida [31], donde se reportan valores de capacidad calórica de $1.98 \mathrm{~J} / \mathrm{gK}$ a $25^{\circ} \mathrm{C}$, mostrando así una diferencia no mayor al $1 \%$ respecto al modelo polinomial evaluado (ec. 9), considerando con ello éste modelo adecuado para la evaluación y extrapolación de valores de capacidad calórica de los biodiesel.

Los modelos matemáticos obtenidos para la evaluación del Número Cetano $(\mathrm{CN})$ a partir de la composición de ácidos grasos muestran una alta relación con lo definido mediante modelos teóricos dados en la literatura, logrando una fiabilidad cercana al $90 \%$ de los modelos conseguidos, permitiendo el uso de estos en el proceso de cálculo de los elementos de calidad en el desarrollo de biocombustibles. El número de datos es en este caso suficiente, más para la generación de un modelo mejor es necesario contar una mayor cantidad de datos, logrando con esto estrechar la diferencia entre los valores generados por otros autores y los valores reportados para los biocombustibles en la literatura.

La Gravedad API evaluada desde la ecuación 16, y en la que se tuvieron en cuenta como valores de densidad, los ya obtenidos desde los modelos Rockett, Rediel y Yamaha \& Gunn, muestra un crecimiento con el incremento de la temperatura lo que es coherente de acuerdo a lo obtenido con Rivera [25] y es consistente con las normas técnicas expuestas en las tablas I y II.

Finalmente se concluye que la metodología empleada en esta investigación ha permitido evidenciar, que los datos resultantes para las propiedades fisicoquímicas a partir de la relación de las ecuaciones y algoritmos evaluados son próximos a los valores experimentales reportados en la bibliografía, permitiendo a partir de este estudio analizar los procesos de producción y sus ajustes a valores reales de referencia.

Así mismo se concluye que las propiedades fisicoquímicas muestran una dependencia con la temperatura, dada ésta desde la respuesta de los modelos a la variación térmica que oscila desde la temperatura ambiente hasta la crítica, cobrado con ello relevancia el análisis y uso de modelos matemáticos teóricos aplicados a la evaluación de las propiedades fisicoquímicas en la producción de biodiesel.

\section{REFERENCIAS}

[1] A. Vivas, "Estudio y Obtención de Biodiesel a partir de Residuos Grasos de Origen Bovino," Universidad Tecnológica de Pereira, 2010.

[2] ICONTEC, "Norma Técnica Colombiana, NTC 5444, Biodiesel para Uso en Motores Diésel Especificaciones," 2006.

[3] ICONTEC, "Norma Técnica Colombiana, NTC 1438, Petróleo y sus Derivados, Combustibles para Motores Diesel en Colombia," 2013.

[4] ICONTEC, "Guía Técnica Colombiana, GTC 213, Guía de Sostenibilidad en las Etapas de Producción y Procesamiento de Biomasa en la Cadena de Biocombustibles en Colombia," 2011.

[5] R. García, "Estudio y simulación del proceso de producción de biodiésel: aplicación a la predicción de las propiedades físicas," Tesis Doctoral, Ingeniería Química y Tecnologías del Medio Ambiente, Universidad de Zaragosa, 2012.

[6] A. Blangino y S. Romano, "Modelado y correlación de propiedades en biodiesel," Anales de la Asociación Física Argentina, vol. 16, pp. 93-97, 2004.

[7] J. Quezada, "Evaluación de la energía de combustión y otros parámetros físico-químicos del biodiesel obtenido a partir de cinco aceites vegetales," Honduras, 2007.

[8] K. Cheenkachorn, "Predicting properties of biodiesels using statistical models and artificial neural networks," As. J. Energy Env, 7, pp. 299-306, 2006. DOI: 10.1.1.451.9146\&rep

[9] P. Benjumea, G. Chaves y C. Vargas, "Efecto de la temperatura sobre la densidad del biodiesel de aceite de palma y sus mezclas con diésel convencional," Energética, 2006.

[10] B. Abdalla, and O. Oshaik, "Base - Transesterification process for biodiesel fuel production from spent frying oil", Agricultural sciences, Vol 4, pp. 85-88, 2013. DOI:10.4236/as.2013.49b015

[11] American Society for Testing and Materials (ASTM), "Standard D6751, Standard Specification for Diesel Fuel Oils," ASTM, West Conshohocken, 2008.

[12] F. Lujaji, Cs. Bereczky, Novak and Mbarawa, "Cetane number and thermal properties of vegetable oil, biodiesel, 1-butanol and diesel blends," Journal of Thermal Analysis and Calorimetry, vol. 102, $\mathrm{n}^{\mathrm{o}} .3$, pp. 1175-1181. ISSN 1572-8943, 2010. DOI:10.1007/s10973-010-0733-9

[13] G. Knothe, A. C. Matheaus and T. W. Ryan, "Cetane numbers of branched and straight-chain fatty esters determined in an ignition quality tester," Fuel, vol. 82, pp. 971-975. ISSN 0016-2361. 11, 2003. DOI:10.1016/s0140-6701(04)91493-2 
[14] I. Sánchez y K. Huertas, "Obtención y caracterización del biodiesel a partir de aceite de semillas de recinus communis. higuerilla, modificadas genéticamente y cultivadas en el eje cafetero", 2012.

[15] P. Castro, J. Coello, y L. Castillo, "Opciones para la producción uso de biodiesel en el Perú," 1a Edición. Lima (Perú): Ediciones Soluciones prácticas, 2007.

[16] T. Yamada and R.D. Gunn, "Saturated Liquid Molar Volumen". The Rackett Equation, J. Chem. Eng. Data: 18, pp. 234-236., 1973

[17] R. C. Reid, J. M. Prausnitz and T. K. Sherwood, "The properties of Gases and Liquids", 4ta Edición, McGraw Hill, 1987.

[18] Riedel, L, Die "Flüssigkeitsdichte im sättigungszustand. untersuchungen über eine erweiterung des theorems der übereinstimmenden zustände," Teil II, Chem. Eng.Tech.: 26, pp. 259-264, 1954. DOI:10.1002/cite.330260504

[19] S. Kerschbaum, and G. Rinke, "Measurement of the temperature dependent viscosity of biodiesel fuels," Fuel, Vol 83, pp. 287-291, 2004. DOI:10.1016/s0140-6701(04)94512-2

[20] S. Channiwala and P. Parikh, "A unified Correlation for Estimating HHV of solid, liquid and gaseous fuel," Fuel, Vol 81, pp. 1051-1063, 2002. DOI:10.1016/s0016-2361(01)00131-4

[21] A. Demirbas, "A realistic fuel alternative for diesel engines," London: Springer-Verlag. ISBN 10: 1846289947, 2008.

[22] B. Valencia, “Termodinámica,” U. N. Manizales, 2011.

[23] K. S. Anand, "A comprehensive approach for estimating thermo-physical properties of biodiesel fuels," Applied Thermal Engineering, Vol 31, pp. 235-242, 2011. DOI:10.1016/j.applthermaleng.2010.09.003

[24] Y. Barroto, R. Rodriguez, L. Pérez and N. Frontela, "Cetane number estimation of biofuels from their fatty acid composition," Ingeniería Mecánica. Vol 15, pp. 147-157, 2012.

[25] A. Rivera, "Cálculo de las propiedades fisicoquímicas del biodiesel y sus mezclas con diésel a partir de reglas de mezclado," Tesis de Grado, Universidad de Cartagena, 2013

[26] B. A. Benavides, "El fraccionamiento por cristalización del biodiesel de aceite de palma como alternativa para mejorar sus propiedades de flujo a baja temperatura,” Rev. Fac. Ing. Univ. Antioquia, 43, pp. 7-17, 2008.

[27] A. H. Yuan, "Predicting the temperature dependent viscosity of biodiesel fuels", 2008. DOI:10.1016/j.fuel.2008.11.011

[28] E.Caballero Moreno, "Viabilidad técnica del biodiesel en Colombia," IQUN, México D.F., 2012.

[29] ICONTEC, “Guía Técnica Colombiana, GTC 213, Guía de Sostenibilidad en las Etapas de Producción y Procesamiento de Biomasa en la Cadena de Biocombustibles en Colombia", 2011.

[30] UNE-EN 14214, Combustibles de automoción. Ésteres metílicos de ácidos grasos (FAME) para motores diésel. Requisitos y métodos de ensayo, 2009.

[31] M. P. Dzida, "The effect of temperature and pressure on the physicochemical properties of petroleum diesel oil and biodiesel fuel," Fuel, 87, pp. 1941-1948, 2008. DOI: 10.1016/j.fuel.2007.10.010

[32] M. Fanguri and D. Clemens, "The effect of mixing on transesterification of beef tallow," Bioresourse Technology 69, 1999. DOI:10.1016/s09608524(98)00184-9

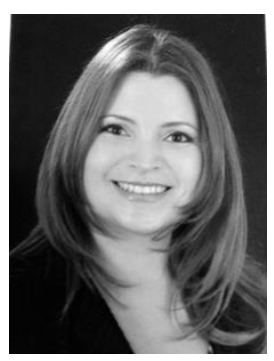

Anny Astrid Espitia Cubillos, nació en Bogotá, Colombia. Es Ingeniera Industrial graduada en 2002 de la Universidad Militar Nueva Granada, Bogotá, Colombia; se graduó como MSc. en Ingeniería Industrial en 2006 de la Universidad de Los Andes, Bogotá, Colombia; actualmente es estudiante de
Doctorado en Ingeniería de la Universidad de Carabobo, Valencia, Venezuela.

Desde 2004 es profesora universitaria. Se vinculó como docente de tiempo completo de Ingeniería Industrial a la Universidad Militar Nueva Granada en el año 2007, donde es profesora asociada desde 2016. Sus intereses investigativos incluyen métodos cuantitativos y responsabilidad social. ORCID: http://orcid.org/0000-0002-4791-0250

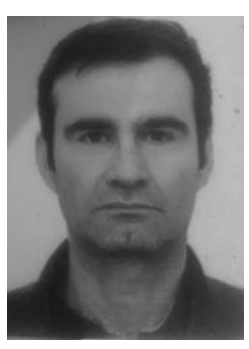

Arnoldo Emilio Delgado Tobón, Ingeniero Mecánico de la Universidad Nacional de Colombia, Bogotá, Colombia y Magister en Ingeniería Mecánica de la Universidad de Los Andes, Bogotá, Colombia.

Docente investigador de la Universidad Militar Nueva Granada, líder del grupo de investigación Energía Alternativa y miembro del grupo de investigación VOLTA, con experiencia investigativa en las áreas de sistemas de conversión de energía, materiales poliméricos y procesos de manufactura, combustibles alternativos, sistemas de gasificación, proyectos agroambientales y de mantenimiento, que se traduce en la participación en eventos, cinco equipos fabricados, tres libros publicados, 61 artículos publicados en revistas indexadas, de los cuales 40 son A1, una patente publicada en la OMPI, tres patentes otorgadas por la SIC, seis patentes publicadas por la SIC y tres en proceso. Ganador del Premio Nacional del Inventor Colombiano año 2015 según la SIC-ACAC-OMPI.\} ORCID: http://orcid.org/0000-0002-8743-8958

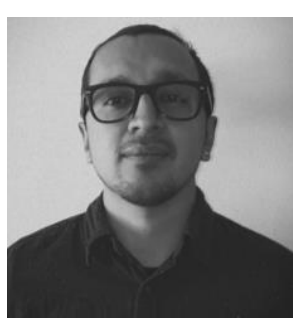

Sergio Alejandro Camargo Vargas, nació en Bogotá, Colombia. Recibió su grado en Ingeniería Catastral y Goedesia en la Universidad Distrital Francisco José de Caldas, Bogotá, en 2011, con estudios en Geofísica en la Universidad Nacional de Colombia y la Universidad de Niza Sophia en Francia, en 2013. Actualmente cursando una Maestría en Tecnologías de la Información Geográfica en la Universidad de Manizales, Caldas, desde 2016.

Desde 2014 hasta 2016, fue coordinador del Sistema Integrado de Análisis Territorial en la Universidad de Caldas y profesor asistente en investigación en la facultad de Ingeniería de la Universidad Nacional, en Manizales, Caldas. Asistente de investigación de la Facultad de Ingeniería de la Universidad Militar Nueva Granada, en Bogotá y, consultor en temas de Data Science y Sistemas de información Geográfica.

ORCID: http://orcid.org/0000-0002-1942-4976 